\title{
Differentiation of neonatal dorsal root ganglion-derived neural stem cells into oligodendrocytes after intrathecal transplantation into a cauda equina lesion model
}

\author{
Z.Y. Fu*, J.G. Shi, N. Liu*, L.S. Jia, W. Yuan and Y. Wang \\ Department of Orthopedics, Changzheng Hospital, \\ Second Military Medical University of China, Shanghai, China \\ *These authors contributed equally to this study. \\ Corresponding author: J.G. Shi \\ E-mail: jiangangshi812@163.com
}

Genet. Mol. Res. 12 (4): 6092-6102 (2013)

Received January 3, 2013

Accepted October 3, 2013

Published December 2, 2013

DOI http://dx.doi.org/10.4238/2013.December.2.7

\begin{abstract}
Cauda equina syndrome (CES) is characterized by varying patterns of low back pain, sciatica, lower extremity sensorimotor loss, and bowel and bladder dysfunction. The prognosis for complete recovery of CES is dependent on not only the time before surgical intervention with decompression but also the severity of the nerve damage. Delayed or severe nerve compression impairs the capability of nerve regeneration. Transplantation of neural stem cells (NSCs) may facilitate axon regeneration and functional recovery in a spectrum of neurological disorders. Our study shows that the NSCs derived from early postnatal dorsal root ganglion (DRG) are able to proliferate to form neurospheres and differentiate into $\mathrm{O}^{+}$oligodendrocytes but not glial fibrillary acidic protein $\left(\mathrm{GFAP}^{+}\right)$astrocytes or $\beta$ III-tubulin ${ }^{+}$ neurons in vitro. After intrathecal transplantation into the lumbar spinal canal stenosis animal model, most of the GFP-expressing NSCs were induced to differentiate into oligodendrocytes in vivo. Although the
\end{abstract}


recovery of sensorimotor function was not significantly improved in rats with transplantation therapy, our results implied that subarachnoid microinjection of NSCs may promote axon regeneration of DRG neurons in the cauda equina model after nerve injury.

Key words: Neonatal dorsal root; Ganglia; Neural stem cells; Cauda equina lesions; Transplantation; Cerebrospinal fluid

\section{INTRODUCTION}

Cauda equina syndrome (CES), most frequently caused by central lumbar disc herniation, is a rare condition characterized by varying symptoms of low back pain, muscle weakness of lower extremities, unilateral or bilateral sciatica, sensory disturbance in saddle area, and loss of visceral function, which results from the compression of the cauda equina (Aho et al., 1969; Gitelmn et al., 2008; Ma et al., 2009). The functional recovery of CES is limited in patients subjected to surgical decompression long after the onset of symptoms, which is attributed to severe nerve damage and the failure of axon regeneration (Gardner et al., 2011).

Transplantation of neural stem cells (NSCs) is a promising therapeutic strategy for many neurological disorders characterized by failure of nervous system endogenous repair mechanisms for restoring tissue damage and rescuing loss of function. NSCs are capable of self-renewal and can give rise to neurons and glia (Reynolds and Weiss, 1992). Under pathological conditions in the central nervous system (CNS), loss of either neural or glial cells disrupts refined neural circuits and their function. The replenishment of neurons by transplantation of NSCs helps to maintain the integrity of the neural circuits required for proper functioning (Pluchino et al., 2005; De Feo et al., 2012). Moreover, the transplanted NSCs stimulate the endogenous repair mechanism via secretion of a plethora of trophic growth factors required for neuroprotection (De Feo et al., 2012). However, the feasibility of stem cell differentiation to neurons after transplantation into the adult lesioned nervous system as well as proper integration into neural circuits and emission of axons reaching their targets is still a matter of contention, because the role of NSCs in functional recovery is unclear (Xu et al., 2010; Garbossa et al., 2012).

In the peripheral nervous system, cauda equina lesions often result in sensory nerve damage of dorsal root ganglia (DRG). The effect of stem cell transplantation on the anatomic integrity and functional restoration of the peripheral nervous system after nerve injury remains elusive. Mesenchymal stem cells promote the survival of DRG neurons in the culture system by inhibiting the metalloproteinase pathway (Scuteri et al., 2011). Adipose-derived stem cells secrete trophic factors and enhance neurite outgrowth of DRG neurons in vitro (Sowa et al., 2012). Moreover, CNS stem/progenitor cells have been shown to survive and differentiate into neurons and peripheral glia after xenotransplantation into adult DRG (Brannvall et al., 2006). Neural crest-derived stem cells have recently been isolated and characterized from DRG and trigeminal ganglia (Namaka et al., 2001; Lagares et al., 2007; Li et al., 2007). Early postnatal DRG contained basic fibroblast growth factor (bFGF)-responsive neuronal precursors, which differentiated into neurons after removal of bFGF and addition of trophic factors (Namaka et al., 2001). Neural precursors isolated from an adult DRG explant culture could differentiate into neurons, glia, and smooth muscle cells (Li et al., 2007). However, the fate of the NSCs 
after intrathecal transplantation into the cerebrospinal fluid around cauda equina lesions has not been demonstrated yet.

In the present study, NSCs isolated from neonatal DRG were used to repair damaged cauda equina in a rat model of lumbar spinal canal stenosis (LCS). Multipotent neonatal DRGNSCs most frequently differentiated into oligodendrocytes in vitro. Furthermore, the NSCs were intrathecally transplanted by subarachnoid microinjection and found to survive in the cauda equina and differentiate into oligodendrocytes.

\section{MATERIAL AND METHODS}

\section{Animal care}

Neonatal and adult male Sprague-Dawley rats weighing 200-250 g were obtained from the Animal Center of the Second Military Medical University of China. The surgical interventions for animal experiments were approved by the Institutional Animal Committee, and the animals were cared for in accordance with the Guide for the Care and Use of Laboratory Animals after the surgery (National Research Council, 1996) and the Guidelines and Policies for Rodent Survival Surgery provided by the Animal Care and Use Committees of the Second Military Medical University of China.

\section{Culture of DRG-NSCs}

DRG were dissected from postnatal day 2 rats, mechanically dissociated in Hank's balanced saline solution, pH 7.4, and seeded in Dulbecco's modified Eagle's medium (DMEM)/ F12 (Invitrogen) supplemented with 2\% B27, $10 \mathrm{ng} / \mathrm{mL}$ epidermal growth factor (EGF), and $10 \mathrm{ng} / \mathrm{mL}$ bFGF. The cells were incubated in a humidified atmosphere with $5 \% \mathrm{CO}_{2}$ at $37^{\circ} \mathrm{C}$. The medium was changed every 3 days. The dissociated DRG cells formed clusters or neurospheres within 1-2 weeks. Afterwards, the neurospheres were collected, mechanically aspirated, and resuspended in the proliferation culture medium every 4 days to reduce the cell heterogeneity by maintaining small spheres (50-100 cells/sphere). After 3 generations of subcloning, the NSCs derived from the neurospheres were subjected to lentivirus transduction.

\section{Labeling of DRG-NSCs}

The NSCs were dissociated from the neurospheres and inoculated on 6-well plates, 1 day before cell transduction. Next, the cells were exposed to lentivirus-containing medium with the addition of $6 \mu \mathrm{g} / \mathrm{mL}$ polybrene. Polybrene was used to promote the transduction efficiency of the lentivirus. The DRG-NSCs transduced with green fluorescent protein (GFP)encoding lentivirus showed green fluorescence $48-72 \mathrm{~h}$ later, and the efficiency of the transduction was calculated using fluorescence microscopy.

\section{Differentiation of DRG-NSCs in vitro}

Before cell seeding, the coverslips were coated overnight with $15 \mu \mathrm{g} / \mathrm{mL}$ polylysine (Sigma). NSCs dissociated from the neurospheres were seeded on the coverslips and cultured 
in the differentiation culture medium without EGF and bFGF but with $1 \%$ fetal bovine serum. The NSCs were then fixed 7 days later for immunofluorescence staining.

\section{Contusion injury and NSC transplantation}

Thirty-six SD rats, 6 weeks old, weighing 200-250 g, were used for surgical intervention. Under anesthesia with intraperitoneal injection of chloral hydrate, laminectomy was performed at the lumbar 4 (L4) level of the vertebra, and a piece of silicone (10 mm long, $1 \mathrm{~mm}$ wide, and $1 \mathrm{~mm}$ thick) was placed under the laminae of the $\mathrm{L} 5-6$ vertebra $(\mathrm{N}=24)$ to produce the LCS animal model. Twelve randomly selected rats were subjected to NSC transplantation, and another 12 rats were used as control. A sham operation was performed with a simple laminectomy but without contusion injury $(\mathrm{N}=12)$.

For transplantation of NSCs, we removed the silicone 7 days after the contusion injury and then intrathecally injected $8 \mu \mathrm{L}$ cell suspension containing approximately 800,000 NSCs (transduced with lentivirus) using a micropulled pipette connected to a Hamilton syringe. As control, $8 \mu \mathrm{L}$ phosphate-buffered saline (PBS) was injected into the subarachnoid space.

\section{Immunohistochemistry assay}

One week after cell transplantation, the rats were perfused with $4 \%$ paraformaldehyde as the fixative. The cauda equina was then extracted, immersed in the same fixative for an additional $3 \mathrm{~h}$, and cryoprotected in a sucrose solution at $4^{\circ} \mathrm{C}$ for 2 days. Then, $15-\mu \mathrm{m}$ thick frozen sections of the cauda equina around the lesion site were prepared longitudinally. For immunostaining, the tissue sections were permeabilized with $0.2 \%$ Triton X-100 and immunoblocked for $30 \mathrm{~min}$ in blocking solution containing goat serum. After rinsing with PBS, the cryostat sections were incubated with primary antibodies against $\beta I I I-t u b u l i n$, glial fibrillary acidic protein (GFAP), or $\mathrm{O} 4$ for $1 \mathrm{~h}$ at $37^{\circ} \mathrm{C}$, followed by incubation with secondary antibodies conjugated to a red fluorophore for $1 \mathrm{~h}$ at $37^{\circ} \mathrm{C}$. The images were photographed by inverted fluorescence microscopy.

\section{Behavioral analysis}

For the tail-flick test, the rats were placed in an acrylic restraint for immobilization 20 min before the test, and the tail was then placed over a slit. A beam of light from a projection lamp (voltage of $18.5 \mathrm{~V}$ ) was focused on the tail skin at the junction between the middle and distal $1 / 3$ of the tail. The latency to respond was recorded with a maximal $15 \mathrm{~s}$ radiant heat stimulus. The test was performed at the time before lesion injury, immediately before cell transplantation, 1 and 7 days after transplantation.

\section{Statistical analysis}

For statistical analysis of the results, the SPSS version 12.0 software, provided by the Statistic Institute of the Second Military Medical University, was used. The effects of the treatment on behavioral function were analyzed using one-way analysis of variance (ANOVA). All results are reported as the means \pm standard error. The significance level was defined at $\mathrm{P}<0.05$. 


\section{RESULTS}

\section{Characterization of NSCs derived from neonatal DRG}

To isolate the neural progenitors from the neonatal rat DRG, we dissociated the DRG into single cells and cultured these cells in proliferation culture medium. Three days later, many cells freely floated in the medium and formed neurospheres in vitro. New clusters could be generated by mechanical dissociation of primary neurospheres followed by culture of the dissociated cells at low density. Cytospun clusters collected from a 2-week suspension culture were immunostained for nestin and p75NTR. Our results showed that $>90 \%$ of the cells in the tertiary neurospheres were labeled by antibodies against nestin and p75NTR (Figure 1).

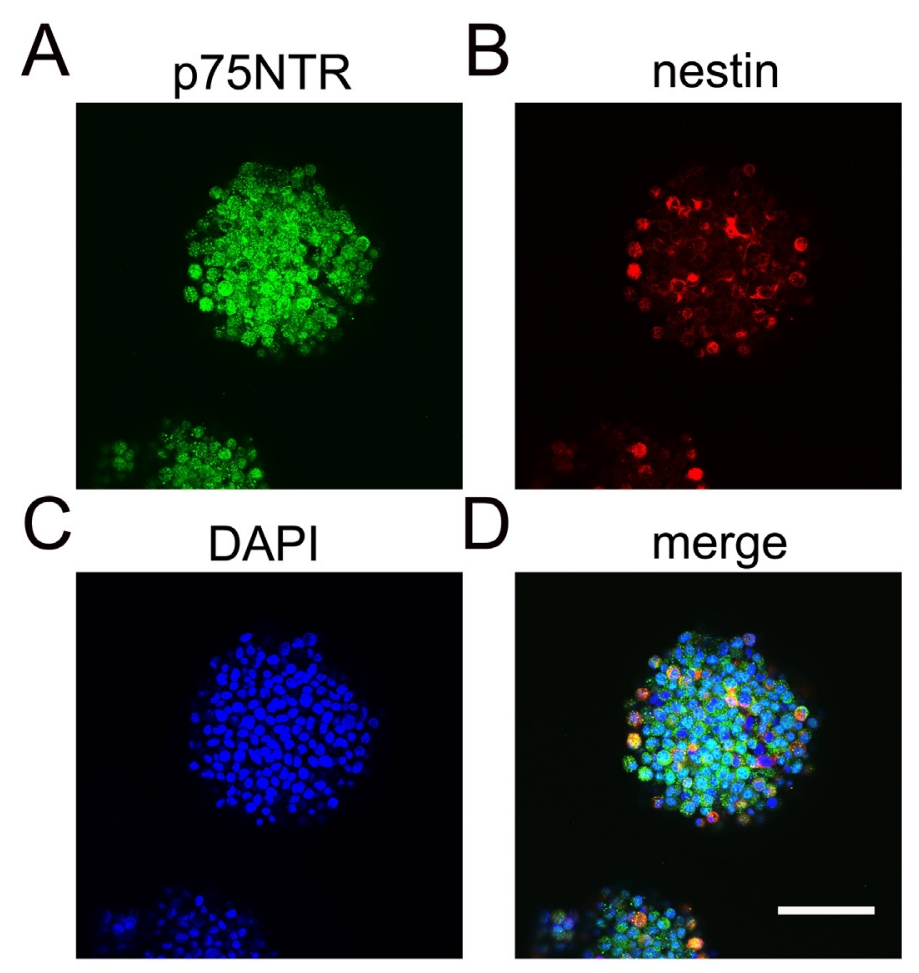

Figure 1. Identification of dorsal root ganglia-neural stem cells (DRG-NSCs). Immunofluorescent staining showed that almost all of NSCs $(>90 \%)$ derived from DRG in the neurospheres were positive for $\mathrm{p} 75 \mathrm{NTR}(\mathbf{A})$ and nestin (B). The total number of cells in the clusters was identified by DAPI staining (C and $\mathbf{D})$. Scale bar $=25 \mu \mathrm{m}$.

The clusters were then infected with lentivirus encoding the easily tracked GFP and cultured in differentiation medium without bFGF and EGF. After 7 days of differentiation in vitro, the neural progenitors in the spheres attached to the surface of the coated coverslips and differentiated into different types of cells. The results of immunostaining showed that $98 \%$ of the GFP-NSCs differentiated into $\mathrm{O}^{+}$oligodendrocytes, $1.4 \%$ into $\beta \mathrm{III}$-tubulin ${ }^{+}$ neurons, and $0.6 \%$ into $\mathrm{GFAP}^{+}$astrocytes (Figure 2). In summary, our data suggested that 
the NSCs derived from neonatal DRG had the capacity of self-renewal and multipotency. Moreover, most of the DRG-NSCs differentiated into oligodendrocytes.

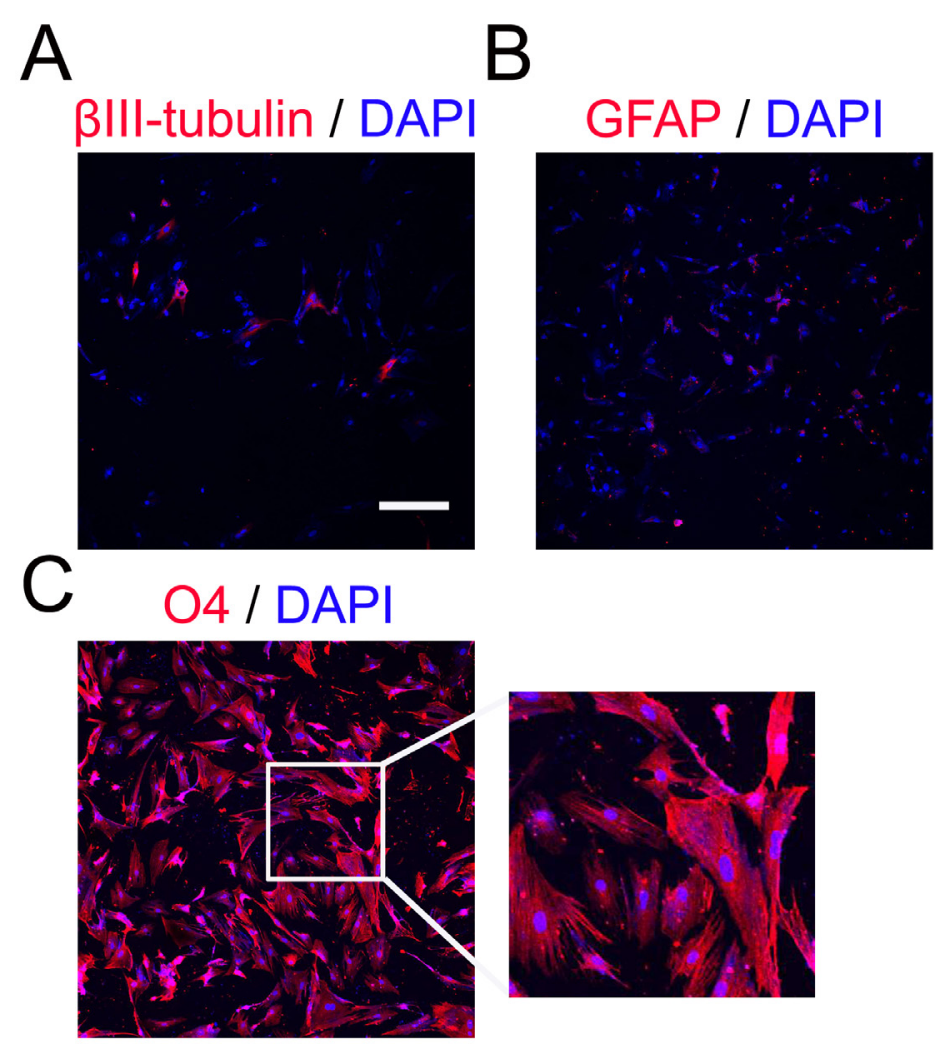

Figure 2. Differentiation of neural stem cells in vitro. The neurospheres were dissociated and cultured in the differentiation culture medium without EGF and bFGF. Immunocytochemistry was performed 7 days later to stain $\beta$ III-tubulin (A), GFAP (B), or O4 (C). The cells were also co-labeled with DAPI. Scale bar $=50 \mu \mathrm{m}$.

\section{Differentiation of DRG-NSCs into oligodendrocytes and their in vivo incorporation into the cauda equina following transplantation}

The neurospheres were dissociated and infected with lentivirus expressing GFP. We found by performing fluorescence-activated cell sorting that $95 \%$ of the NSCs were GFP-positive 3 days after lentivirus transduction (Figure 3A). Approximately 800,000 dissociated GFP-NSCs were intrathecally injected into the cerebrospinal fluid of rats that had been subjected to a cauda equina lesion for 7 days. The cauda equina was cryostatsectioned longitudinally 7 days after the transplantation. To examine the viability of the transplanted cells in the injured cauda equina, the frozen sections were imaged by confocal microscopy. We found that the GFP-positive grafted cells were incorporated into the cauda equina (Figure 3B). 

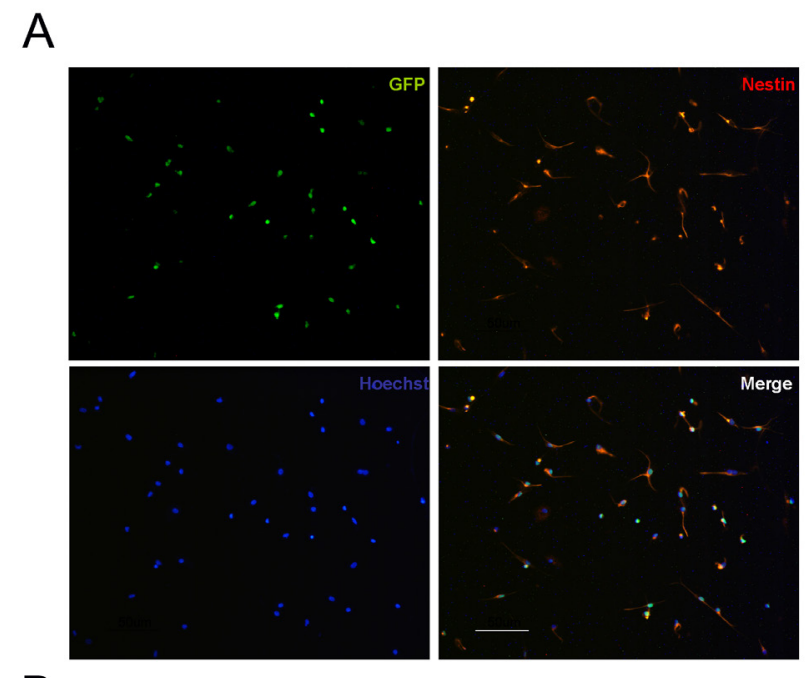

B

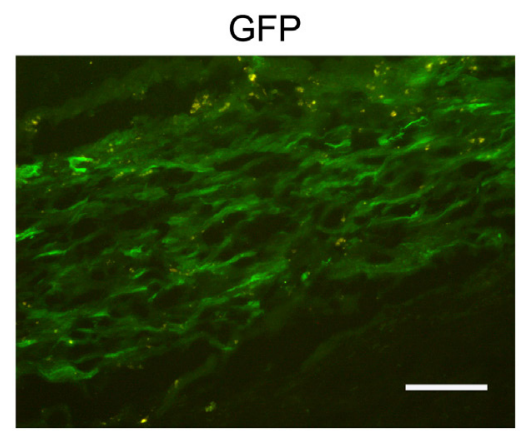

Figure 3. Viral transduction and transplantation of neural stem cells (NSCs) in the cerebrospinal fluid surrounding cauda equina lesions. A. The NSCs were dissociated, transducted with lentivirus and immunostained for GFP, nestin and Hoechst after viral transduction in vitro. Scale bar $=50 \mu \mathrm{m}$. B. The NSCs derived from DRG were transducted with GFP-encoding lentivirus and intrathecally transplanted into the cerebrospinal fluid. Fluorescent images showed that the grafted NSCs survived in the cerebrospinal fluid surrounding cauda equina lesions in vivo 7 days after transplantation. Scale bar $=50 \mu \mathrm{m}$.

To determine the fate of these GFP-positive transplanted cells, we performed immunostaining of the sagittal sections of the cauda equina with antibodies against $\beta$ III-tubulin, GFAP, and O4. The results showed that almost all of the GFP-positive cells co-labeled with $\mathrm{O} 4$ but not with $\beta \mathrm{III}$-tubulin or GFAP, suggesting that the grafted NSCs differentiated into oligodendrocytes but not neurons or astrocytes in vivo (Figure 4).

\section{Assessment of functional recovery following DRG-NSC transplantation}

To evaluate the neuroregenerative activities of the transplanted DRG-derived NSCs, we examined the functional recovery of sensorimotor reflexes using the tail-flick test. Seven days after transplantation, both the experimental group that had received NSCs and the control group that had not received NSCs showed a significant increase in tail-flick latency compared 
with the sham-operated group. There was no significant difference between the experimental group and the control group (Figure 5).

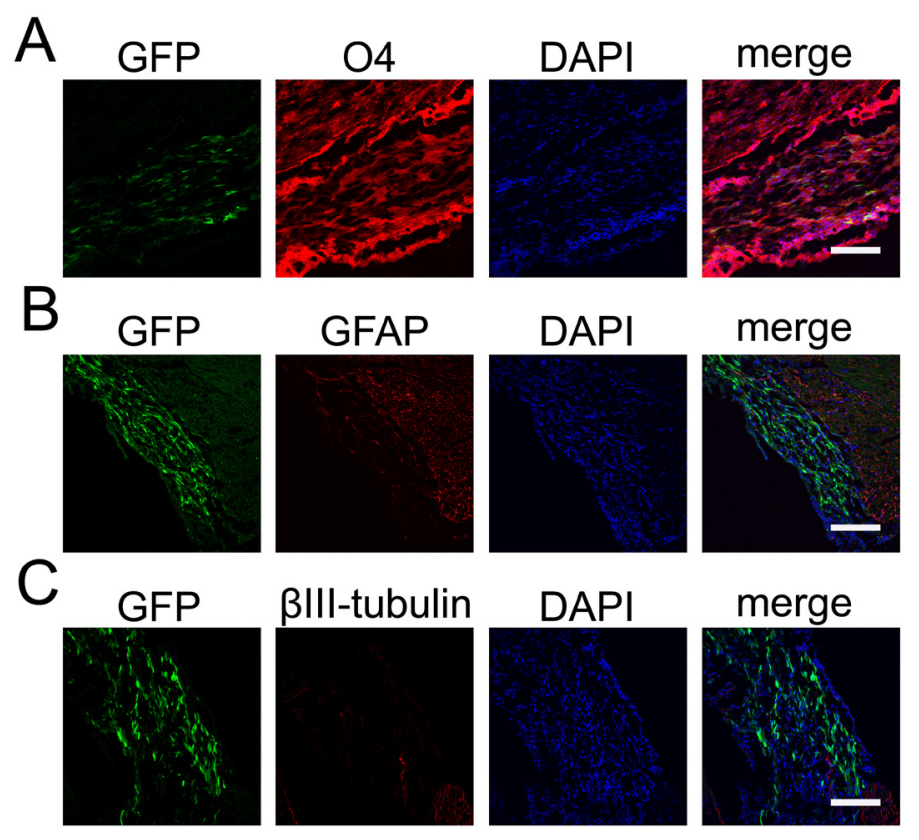

Figure 4. Differentiation of neural stem cells (NSCs) in vivo. The cryostat sections of cauda equina in the lesion site were immunostained 7 days after NSC transplantation. Immunofluorescent staining showed that most of GFPpositive NSCs grafted in the cauda equina differentiated into $\mathrm{O}^{+}$oligodendrocytes $(\mathbf{A})$ but not $\mathrm{GFAP}^{+}$astrocytes (B) or $\beta$ III-tubulin ${ }^{+}$neurons (C). Scale bar $=50 \mu \mathrm{m}$.

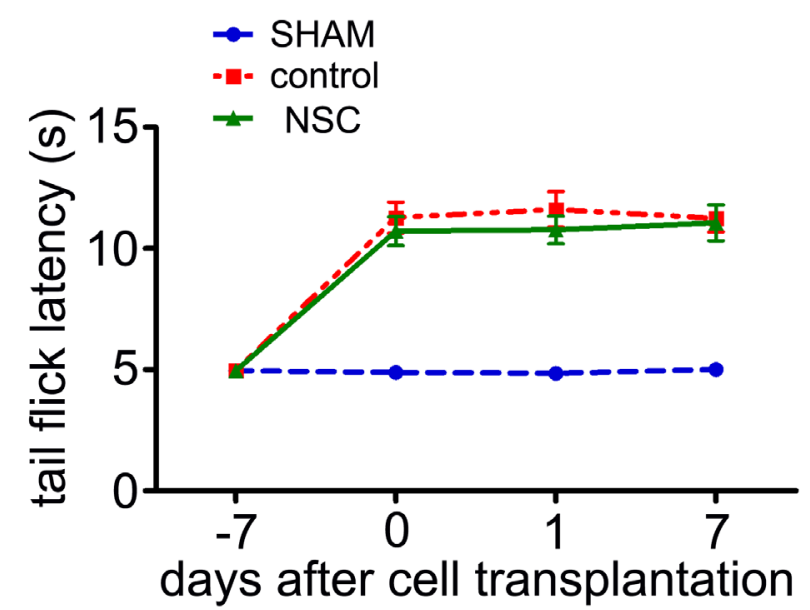

Figure 5. Sensory function of neural stem cell (NSC) transplantation. The sensory function was not improved by NSC transplantation. The LCS rats showed impaired nociceptive capacity compared to the sham-operated group. However, there was no significant difference between the control group and the experimental group, which received NSC transplantation in the tail-flick test. Data are reported as means \pm SE. 


\section{DISCUSSION}

The cerebrospinal fluid, which contacts apical neural progenitors at the ventricular surface and bathes the apical complex of these cells, provides growth- and survival-promoting cues for neural progenitors in the developing and adult brain. The embryonic cerebrospinal fluid is a rich and dynamic source of growth factors for the brain, producing and distributing diffusible signals such as FGF, insulin-like growth factor, sonic hedgehog (Shh), and retinoic acid. Therefore, the embryonic cerebrospinal fluid forms an integral component of the embryonic stem cell niche (Lehtinen and Walsh, 2011). The active role of the cerebrospinal fluid in the adult CNS, especially in the adult spinal cord and cauda equina, is still unclear. Our results showed that the growth factors in the cerebrospinal fluid of the spinal cord and cauda equina instructed the grafted DRG-NSC to differentiate into oligodendrocytes.

NSC transplantation is a promising strategy for the treatment of spinal cord injury. The therapeutic benefits are derived from a number of different mechanisms, from cell replacement to neuroprotection (Fischer, 2000; Llado et al., 2004; De Feo et al., 2012). Accumulating evidence shows that transplanted NSCs survive and differentiate to achieve CNS functional recovery (Cummings et al., 2005; Muraoka et al., 2006; Tarasenko et al., 2007). However, the fate of grafted NSCs in the host remains controversial.

The cerebrospinal fluid plays an important role in the development of neuroectodermal cells and in the survival, proliferation, and fate of NSCs in the adult brain. The cerebrospinal fluid has been reported to induce differentiation of human bone marrow mesenchymal stem cells into neural-like cells (Ye et al., 2011). Moreover, the presence of cerebrospinal fluid in the culture medium enhanced the survival and differentiation of fetal rat NSCs and adult human neural progenitor cells. After differentiation in the cerebrospinal fluid, more GFAP $^{+}$ astroglial cells and less $\beta$ III-tubulin ${ }^{+}$neuronal cells were found in vitro compared with the differentiation of NSCs in a standard culture medium (Buddensiek et al., 2009, 2010).

The intrathecally grafted NSCs survive well in the host spinal cord for as long as 8 months after transplantation and differentiate into astrocytes and oligodendrocytes in vivo (Wu et al., 2002). Here, we showed that NSCs derived from the neonatal rat DRG differentiated into oligodendrocytes in vitro. After transplantation into the cerebrospinal fluid, DRG-NSCs survived and differentiated into oligodendrocytes in the cerebrospinal fluid surrounding the cauda equina lesions. Our results suggested that the host cerebrospinal fluid might provide a suitable microenvironment for differentiation of grafted NSCs into oligodendrocytes.

Previous studies showed that Shh signaling is required for the fate determination of oligodendrocytes from NSCs in the developing brain for promoting oligodendrogenesis via transcriptional activation of Olig1 and Olig2 (Orentas et al., 1999; Nery et al., 2001; Rivera et al., 2010). Bone morphogenic protein signaling enhances astrogenesis at the expense of oligodendrogenesis through upregulation of Id 2 and Id4 to block the nuclear translocation of Olig1 and Olig2 (Kondo and Raff, 2000). In the animal model of experimental allergic encephalomyelitis, nerve growth factor promoted the growth and differentiation of oligodendrocytes to protect neuronal injury. Moreover, FGFR-1 and FGFR-2 drive oligodendrocytes and motor neuron specification during development. In particular, FGFR-1 determines oligodendroglial and neuronal cell fate, whereas FGFR-2 is related to oligodendrocyte specification. Interestingly, these receptors are differentially expressed in different neurogenic progenitors (Galvez-Gontreras et al., 2012). Therefore, we hypothesize that the cerebrospinal fluid in the 
spine contains many more activators of oligodendrogenesis, e.g., Shh, nerve growth factor, and Noggin, than inhibitory growth factors, including bone morphogenic protein, Notch, and Wnt. In contrast, DRG-NSCs might express high levels of FGFR-2.

Oligodendrocytes are particularly susceptible to the toxicity of the acute lesion environment after spinal cord contusion. Loss of oligodendrocytes causes demyelination, impairs the conductive capacity of sensory nerves, and hampers the functional recovery of damaged spinal cords. Replenishment of myelin-forming cells by stem cell transplantation potentially helps to improve myelination and enhances functional recovery from spinal cord injury (Warden et al., 2001; Kocsis et al., 2002). However, we did not find any significant improvement in the animal model of LCS with transplanted NSCs, despite the differentiation of the NSCs into oligodendrocytes. First, the replenished oligodendrocytes might not have sufficient time to remyelinate. Our preliminary experiments showed that in vitro formation of myelin from oligodendrocytes in the dorsal root axons required 5 weeks. Secondly, the newly differentiated oligodendrocytes were extinguished by the immune cells due to allograft rejection. We also found that the liver, spleen, and other organs were enlarged after transplantation. Our further efforts will focus on the application of immunosuppressive agents (tacrolimus) to prolong the viability of transplanted cells and recover the deficits of sensorimotor function.

\section{ACKNOWLEDGMENTS} \#30872613).

Research supported by the National Natural Science Foundation of China (grant

\section{REFERENCES}

Aho AJ, Auranen A and Pesonen K (1969). Analysis of cauda equina symptoms in patients with lumbar disc prolapse. Preoperative and follow-up clinical and cystometric studies. Acta Chir. Scand. 135: 413-420.

Brannvall K, Sandelin M, Wallenquist U, Forsberg-Nilsson K, et al. (2006). Central nervous system stem/progenitor cells form neurons and peripheral glia after transplantation to the dorsal root ganglion. Neuroreport 17: 623-628.

Buddensiek J, Dressel A, Kowalski M, Storch A, et al. (2009). Adult cerebrospinal fluid inhibits neurogenesis but facilitates gliogenesis from fetal rat neural stem cells. J. Neurosci. Res. 87: 3054-3066.

Buddensiek J, Dressel A, Kowalski M, Runge U, et al. (2010). Cerebrospinal fluid promotes survival and astroglial differentiation of adult human neural progenitor cells but inhibits proliferation and neuronal differentiation. $B M C$ Neurosci. 11: 48.

Cummings BJ, Uchida N, Tamaki SJ, Salazar DL, et al. (2005). Human neural stem cells differentiate and promote locomotor recovery in spinal cord-injured mice. Proc. Natl. Acad. Sci. U. S. A. 102: 14069-14074.

De Feo D, Merlini A, Laterza C and Martino G (2012). Neural stem cell transplantation in central nervous system disorders: from cell replacement to neuroprotection. Curr. Opin. Neurol. 25: 322-333.

Fischer I (2000). Candidate cells for transplantation into the injured CNS. Prog. Brain Res. 128: 253-257.

Galvez-Contreras AY, Gonzalez-Castaneda RE, Luquin S and Gonzalez-Perez O (2012). Role of fibroblast growth factor receptors in astrocytic stem cells. Curr. Signal. Transduct. Ther. 7: 81-86.

Garbossa D, Boido M, Fontanella M, Fronda C, et al. (2012). Recent therapeutic strategies for spinal cord injury treatment: possible role of stem cells. Neurosurg. Rev. 35: 293-311.

Gardner A, Gardner E and Morley T (2011). Cauda equina syndrome: a review of the current clinical and medico-legal position. Eur. Spine J. 20: 690-697.

Gitelman A, Hishmeh S, Morelli BN, Joseph SA Jr, et al. (2008). Cauda equina syndrome: a comprehensive review. Am. J. Orthop. 37: 556-562.

Kocsis JD, Akiyama Y, Lankford KL and Radtke C (2002). Cell transplantation of peripheral-myelin-forming cells to repair the injured spinal cord. J. Rehabil. Res. Dev. 39: 287-298.

Kondo T and Raff M (2000). The Id4 HLH protein and the timing of oligodendrocyte differentiation. EMBO J. 19: 1998-2007. 
Lagares A, Li HY, Zhou XF and Avendano C (2007). Primary sensory neuron addition in the adult rat trigeminal ganglion: evidence for neural crest glio-neuronal precursor maturation. J. Neurosci. 27: 7939-7953.

Lehtinen MK and Walsh CA (2011). Neurogenesis at the brain-cerebrospinal fluid interface. Annu. Rev. Cell Dev. Biol. 27: 653-679.

Li HY, Say EH and Zhou XF (2007). Isolation and characterization of neural crest progenitors from adult dorsal root ganglia. Stem Cells 25: 2053-2065.

Llado J, Haenggeli C, Maragakis NJ, Snyder EY, et al. (2004). Neural stem cells protect against glutamate-induced excitotoxicity and promote survival of injured motor neurons through the secretion of neurotrophic factors. Mol. Cell Neurosci. 27: 322-331.

Ma B, Wu H, Jia LS, Yuan W, et al. (2009). Cauda equina syndrome: a review of clinical progress. Chin. Med. J. 122: 1214-1222.

Muraoka K, Shingo T, Yasuhara T, Kameda M, et al. (2006). The high integration and differentiation potential of autologous neural stem cell transplantation compared with allogeneic transplantation in adult rat hippocampus. Exp. Neurol. 199: 311-327.

Namaka MP, Sawchuk M, MacDonald SC, Jordan LM, et al. (2001). Neurogenesis in postnatal mouse dorsal root ganglia. Exp. Neurol. 172: 60-69.

Nery S, Wichterle H and Fishell G (2001). Sonic hedgehog contributes to oligodendrocyte specification in the mammalian forebrain. Development 128: 527-540.

Orentas DM, Hayes JE, Dyer KL and Miller RH (1999). Sonic hedgehog signaling is required during the appearance of spinal cord oligodendrocyte precursors. Development 126: 2419-2429.

Pluchino S, Zanotti L, Deleidi M and Martino G (2005). Neural stem cells and their use as therapeutic tool in neurological disorders. Brain Res. Brain Res. Rev. 48: 211-219.

Reynolds BA and Weiss S (1992). Generation of neurons and astrocytes from isolated cells of the adult mammalian central nervous system. Science 255: 1707-1710.

Rivera FJ, Steffenhagen C, Kremer D, Kandasamy M, et al. (2010). Deciphering the oligodendrogenic program of neural progenitors: cell intrinsic and extrinsic regulators. Stem Cells Dev. 19: 595-606.

Scuteri A, Ravasi M, Pasini S, Bossi M, et al. (2011). Mesenchymal stem cells support dorsal root ganglion neurons survival by inhibiting the metalloproteinase pathway. Neuroscience 172: 12-19.

Sowa Y, Imura T, Numajiri T, Nishino K, et al. (2012). Adipose-derived stem cells produce factors enhancing peripheral nerve regeneration: influence of age and anatomic site of origin. Stem Cells Dev. 21: 1852-1862.

Tarasenko YI, Gao J, Nie L, Johnson KM, et al. (2007). Human fetal neural stem cells grafted into contusion-injured rat spinal cords improve behavior. J. Neurosci. Res. 85: 47-57.

Warden P, Bamber NI, Li H, Esposito A, et al. (2001). Delayed glial cell death following wallerian degeneration in white matter tracts after spinal cord dorsal column cordotomy in adult rats. Exp. Neurol. 168: 213-224.

Wu S, Suzuki Y, Noda T, Bai H, et al. (2002). Immunohistochemical and electron microscopic study of invasion and differentiation in spinal cord lesion of neural stem cells grafted through cerebrospinal fluid in rat. J. Neurosci. Res. 69: 940-945.

Xu L, Xu CJ, Lu HZ, Wang YX, et al. (2010). Long-term fate of allogeneic neural stem cells following transplantation into injured spinal cord. Stem Cell Rev. 6: 121-136.

Ye Y, Zeng YM, Wan MR and Lu XF (2011). Induction of human bone marrow mesenchymal stem cells differentiation into neural-like cells using cerebrospinal fluid. Cell Biochem. Biophys. 59: 179-184. 